\title{
Supporting Information: Spectrally resolving coherent TERS spectroscopy of electrically biased carbon-coated fibers
}

Sergey S. Kharintsev ${ }^{1 *}$, Svetlana V. Saparina ${ }^{1}$, Alexandr I. Fishman ${ }^{1}$, Andrei A. Stolov ${ }^{2}$ and ${\mathrm{Jie} \mathrm{Li}^{2}}^{2}$

${ }^{1}$ Department of Optics and Nanophotonics, Institute of Physics, Kazan Federal University, Kremlevskaya, 16, Kazan, 420008, Russia

${ }^{2}$ OFS, 55 Darling Drive, Avon, CT, 06001, United States

*To whom correspondence should be addressed. E-mail: (S.S.K.) Sergey.Kharintsev@kpfu.ru

The surface dc electrical conductivity at different temperatures was probed in a contact mode by applying voltage to the sample with the help of the atomic force microscopy (AFM) microscope NTEGRA PRIMA ${ }^{\mathrm{TM}}$ (NT-MDT). As a probe, we used conducting AFM cantilevers CSG1-/Pt (NTMDT). The distance between the AFM cantilever, referred to as the working electrode, and a counter electrode connected to upper surface was about $2 \mathrm{~mm}$. During the measurements, the tipsample force was typically in the range of $10-15 \mathrm{nN}$. All the dc conductivity measurements were performed in ambient air. The relative humidity in the lab was in the range $30-50 \%$. 

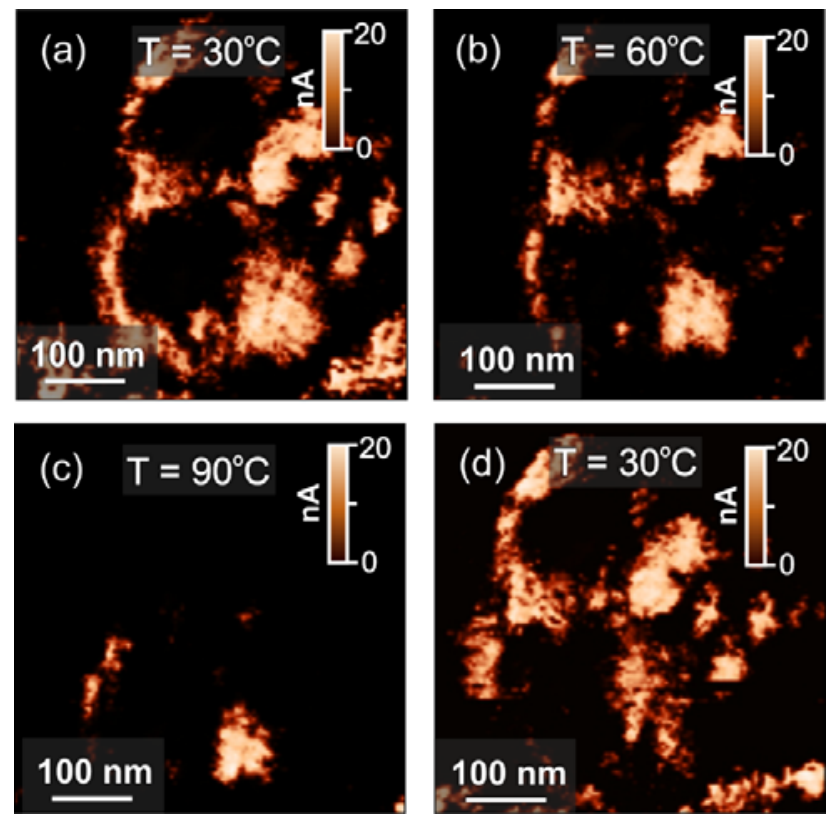

Figure S1. Dc conductivity map of the $31 \mathrm{~nm}$ a-C coating at different temperatures: (a) $30^{\circ} \mathrm{C}$, (b) $60^{\circ} \mathrm{C}$, (c) $90^{\circ} \mathrm{C}$ and (d) $30^{\circ} \mathrm{C}$ after cooling down from $90^{\circ} \mathrm{C}$.

Thermal measurements were performed with an SThM setup (AU040 unit, NT-MDT) using a specialized SThM_P probe (NT-MDT). A tip apex of the probe was a NiCr/Pd resistor with a curvature radius of $<100 \mathrm{~nm}$. The instrumental error was of the order of $0.1{ }^{\circ} \mathrm{C}$.

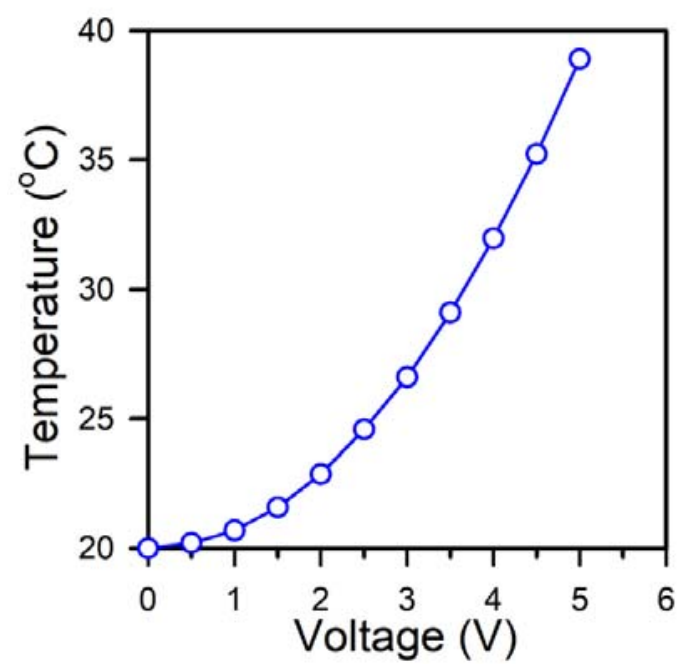


Figure S2. The temperature vs. applied voltage for the $31 \mathrm{~nm}$ a-C coating measured by using scanning thermal microscopy.

Numerical decomposition of anti-Stokes and Stokes bands into elementary peaks were performed using the regularized least squares method. To provide a robust solution, a noisy Raman spectrum was preliminarily smoothened in terms of a singular value decomposition method. All fitted profiles are assumed to be Lorentzian functions. First, the unresolved Stokes band is fitted with the Lorentzian peaks by optimizing their peak positions, full widths at half height and the intensities. The sought parameters of the Stokes peaks were further used as starting values for resolving the anti-Stokes band. The correlation coefficients for the Stokes and anti-Stokes cases are estimated to be 0.985 and 0.980 , respectively. A multi-peak fitting analysis displays seven elementary bands: $G\left(1550 \mathrm{~cm}^{-1}\right), D\left(1330 \mathrm{~cm}^{-1}\right), D^{*}\left(1480 \mathrm{~cm}^{-1}\right), D^{\prime}\left(1620 \mathrm{~cm}^{-1}\right), D^{\prime}\left(1130 \mathrm{~cm}^{-1}\right)$ , $D^{-}\left(1260 \mathrm{~cm}^{-1}\right)$ and $D^{+}\left(1400 \mathrm{~cm}^{-1}\right)$.

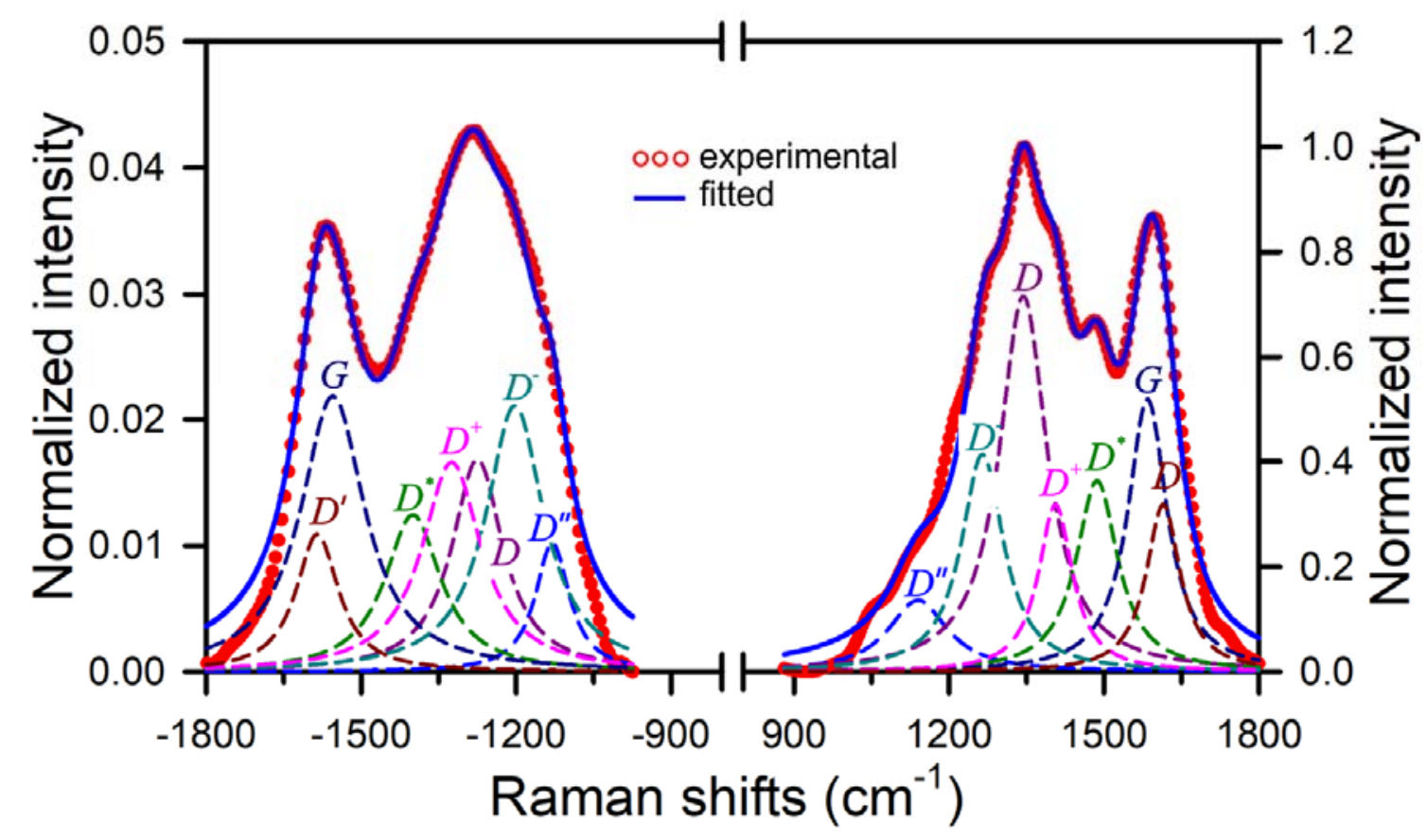

Figure S3. Deconvolution of anti-Stokes and Stokes far-field Raman spectra at zero voltage. 